\title{
Strong-coupling expansion for the two-species Bose-Hubbard model
}

\author{
M. Iskin \\ Department of Physics, Koç University, Rumelifeneri Yolu, 34450 Sariyer, Istanbul, Turkey \\ (Received 16 January 2010; revised manuscript received 9 June 2010; published 30 September 2010)
}

\begin{abstract}
To analyze the ground-state phase diagram of Bose-Bose mixtures loaded into $d$-dimensional hypercubic optical lattices, we perform a strong-coupling power-series expansion in the kinetic energy term (plus a scaling analysis) for the two-species Bose-Hubbard model with onsite boson-boson interactions. We consider both repulsive and attractive interspecies interaction, and obtain an analytical expression for the phase boundary between the incompressible Mott insulator and the compressible superfluid phase up to third order in the hoppings. In particular, we find a re-entrant quantum phase transition from paired superfluid (superfluidity of composite bosons, i.e., Bose-Bose pairs) to Mott insulator and again to a paired superfluid in all one, two, and three dimensions, when the interspecies interaction is sufficiently large and attractive. We hope that some of our results could be tested with ultracold atomic systems.
\end{abstract}

DOI: 10.1103/PhysRevA.82.033630

PACS number(s): 67.85.-d, 03.75.-b, 37.10.Jk

\section{INTRODUCTION}

The single-species Bose-Hubbard $(\mathrm{BH})$ model is the bosonic generalization of the Hubbard model, and was introduced originally to describe ${ }^{4} \mathrm{He}$ in porous media or disordered granular superconductors [1]. For hypercubic lattices in all dimensions $d$, there are only two phases in this model: an incompressible Mott insulator at commensurate (integer) fillings and a compressible superfluid phase otherwise. The superfluid phase is well described by weak-coupling theories, but the insulating phase is a strong-coupling phenomenon that only appears when the system is on a lattice. Transition from the Mott insulator to the superfluid phase occurs as the hopping, particle-particle interaction, or the chemical potential is varied [1].

It is the recent observation of this transition in effectively three- [2], one- [3], and two-dimensional [4,5] optical lattices, which has been considered one of the most remarkable achievements in the field of ultracold atomic gases, since it paved the way for studying other strongly correlated phases in similar setups. Such lattices are created by the intersection of laser fields, and they are nondissipative periodic potential energy surfaces for the atoms. Motivated by this success in experimentally simulating the single-species $\mathrm{BH}$ model with ultracold atomic Bose gases loaded into optical lattices, there has been recently an intense theoretical activity in analyzing $\mathrm{BH}$ as well as Fermi-Hubbard type models [6].

For instance, in addition to the Mott insulator and singlespecies superfluid phases, it has been predicted that the two-species $\mathrm{BH}$ model has at least two additional phases: an incompressible supercounter flow and a compressible paired superfluid phase [7-16]. Our main interest here is in the latter phase, where a direct transition from the Mott insulator to the paired superfluid phase (superfluidity of composite bosons, i.e., Bose-Bose pairs) has been predicted, when both species have integer fillings and the interspecies interaction is sufficiently large and attractive. Given that the interspecies interactions can be fine tuned in ongoing experiments (e.g., with ${ }^{41} \mathrm{~K}-{ }^{87} \mathrm{Rb}$ with mixtures $[17,18]$ ), via using Feshbach resonances, we hope that some of our results could be tested with ultracold atomic systems.

In this paper, we examine the ground-state phase diagram of the two-species $\mathrm{BH}$ model with onsite boson-boson interactions in $d$-dimensional hypercubic lattices, including both the repulsive and attractive interspecies interaction, via a strong-coupling perturbation theory in the hopping. We carry the expansion out to third order in the hopping, and perform a scaling analysis using the known critical behavior at the tip of the insulating lobes, which allows us to accurately predict the critical point, and the shape of the insulating lobes in the plane of the chemical potential and the hopping. This technique was previously used to discuss the phase diagram of the single-species BH model [19-23], the extended BH model [24], and the hardcore $\mathrm{BH}$ model with a superlattice [25], and its results showed an excellent agreement with Monte Carlo simulations [23,25]. Motivated by the success of this technique with these models, here we apply it to the two-species $\mathrm{BH}$ model, hoping to develop an analytical approach which could be as accurate as the numerical ones.

The remaining paper is organized as follows. After introducing the model Hamiltonian in Sec. II, we develop the strong-coupling expansion in Sec. III, where we derive an analytical expression for the phase boundary between the incompressible Mott insulator and the compressible superfluid phase. Then, in Sec. IV, we propose a chemical-potential extrapolation technique based on scaling theory to extrapolate our third-order power-series expansion into a functional form that is appropriate for the Mott lobes, and use it to obtain typical ground-state phase diagrams. A brief summary of our conclusions is given in Sec. V.

\section{TWO-SPECIES BOSE-HUBBARD MODEL}

To describe Bose-Bose mixtures loaded into optical lattices, we consider the following two-species BH Hamiltonian,

$$
\begin{aligned}
H= & -\sum_{i, j, \sigma} t_{i j, \sigma} b_{i, \sigma}^{\dagger} b_{j, \sigma}+\sum_{i, \sigma} \frac{U_{\sigma \sigma}}{2} \widehat{n}_{i, \sigma}\left(\widehat{n}_{i, \sigma}-1\right) \\
& +U_{\uparrow \downarrow} \sum_{i} \widehat{n}_{i, \uparrow} \widehat{n}_{i, \downarrow}-\sum_{i, \sigma} \mu_{\sigma} \widehat{n}_{i, \sigma},
\end{aligned}
$$

where the pseudospin $\sigma \equiv\{\uparrow, \downarrow\}$ labels the trapped hyperfine states of a given species of bosons, or labels different types of bosons in a two-species mixture, $t_{i j, \sigma}$ is the tunneling (or hopping) matrix between sites $i$ and $j, b_{i, \sigma}^{\dagger}\left(b_{i, \sigma}\right)$ is the 
boson creation (annihilation) and $\widehat{n}_{i, \sigma}=b_{i, \sigma}^{\dagger} b_{i, \sigma}$ is the boson number operator at site $i, U_{\sigma \sigma^{\prime}}$ is the strength of the onsite boson-boson interaction between $\sigma$ and $\sigma^{\prime}$ components, and $\mu_{\sigma}$ is the chemical potential. In this manuscript, we consider a $d$-dimensional hypercubic lattice with $M$ sites, for which we assume $t_{i j, \sigma}$ is a real symmetric matrix with elements $t_{i j, \sigma}=t_{\sigma} \geqslant 0$ for $i$ and $j$ nearest neighbors and 0 otherwise. The lattice coordination number (or the number of nearest neighbors) for such lattices is $z=2 d$.

We take the intraspecies interactions to be repulsive $\left(\left\{U_{\uparrow \uparrow}, U_{\downarrow \downarrow}\right\}>0\right)$, but discuss both repulsive and attractive interspecies interaction $U_{\uparrow \downarrow}$ as long as $U_{\uparrow \uparrow} U_{\downarrow \downarrow}>U_{\uparrow \downarrow}^{2}$. This guarantees the stability of the mixture against collapse when $U_{\uparrow \downarrow} \ll 0$, and against phase separation when $U_{\uparrow \downarrow} \gg 0$. However, when the interspecies interaction is sufficiently large and attractive, we note that instead of a direct transition from the Mott insulator to a single-particle superfluid phase, it is possible to have a transition from the Mott insulator to a paired superfluid phase (superfluidity of composite bosons, i.e., Bose-Bose pairs) [7-16]. Therefore, one needs to consider both possibilities, as discussed next.

\section{STRONG-COUPLING EXPANSION}

We use the many-body version of the Rayleigh-Schrödinger perturbation theory in the kinetic energy term to perform the expansion (in powers of $t_{\uparrow}$ and $t_{\downarrow}$ ) for the different energies needed to carry out our analysis. The strong-coupling expansion technique was previously used to discuss the phase diagram of the single-species $\mathrm{BH}$ model [19-21,23], the extended BH model [24], and the hardcore BH model with a superlattice [25], and its results showed an excellent agreement with Monte Carlo simulations [23,25]. Motivated by the success of this technique with these models, here we apply it to the two-species BH model.

To determine the phase boundary separating the incompressible Mott phase from the compressible superfluid phase within the strong-coupling expansion method, one needs the energy of the Mott phase and of its "defect" states (those states which have exactly one extra elementary particle or hole about the ground state) as a function of $t_{\uparrow}$ and $t_{\downarrow}$. At the point where the energy of the incompressible state becomes equal to its defect state, the system becomes compressible, assuming that the compressibility approaches zero continuously at the phase boundary. Here, we remark that this technique cannot be used to calculate the phase boundary between two compressible phases.

\section{A. Ground-state wave functions}

The perturbation theory is performed with respect to the ground state of the system when $t_{\uparrow}=t_{\downarrow}=0$, and therefore we first need zeroth-order wave functions of the Mott phase and of its defect states. To zeroth order in $t_{\uparrow}$ and $t_{\downarrow}$, the Mott insulator wave function can be written as

$$
\left|\Psi_{\text {Mott }}^{\mathrm{ins}(0)}\right\rangle=\frac{1}{\sqrt{n_{\uparrow} ! n_{\downarrow} !}} \prod_{i}\left(b_{i, \uparrow}^{\dagger}\right)^{n_{\uparrow}}\left(b_{i, \downarrow}^{\dagger}\right)^{n_{\downarrow}}|0\rangle,
$$

where $\left\langle\widehat{n}_{i, \sigma}\right\rangle=n_{\sigma}$ is an integer number corresponding to the ground-state occupancy of the pseudospin $\sigma$ bosons, $\langle\cdots\rangle$ is the thermal average, and $|0\rangle$ is the vacuum state. On the other hand, the wave functions of the defect states are determined by degenerate perturbation theory. The reason for that lies in the fact that when exactly one extra elementary particle or hole is added to the Mott phase, it could go to any of the $M$ lattice sites, since all of those states share the same energy when $t_{\uparrow}=t_{\downarrow}=0$. Therefore, the initial degeneracy of the defect states is of order $M$.

When the elementary excitations involve a single- $\sigma$-particle (exactly one extra pseudospin $\sigma$ boson) or a single- $\sigma$-hole (exactly one less pseudospin $\sigma$ boson), this degeneracy is lifted at first order in $t_{\uparrow}$ and $t_{\downarrow}$. The treatment for this case is very similar to the single-species BH model [19,24], and the wave functions (to zeroth order in $t_{\uparrow}$ and $t_{\downarrow}$ ) for the single- $\sigma$-particle and single- $\sigma$-hole defect states turn out to be

$$
\begin{gathered}
\left|\Psi_{\mathrm{def}}^{\mathrm{s} \sigma \mathrm{p}(0)}\right\rangle=\frac{1}{\sqrt{n_{\sigma}+1}} \sum_{i} f_{i}^{\mathrm{s} \sigma \mathrm{p}} b_{i, \sigma}^{\dagger}\left|\Psi_{\mathrm{Mott}}^{\mathrm{ins}(0)}\right\rangle, \\
\left|\Psi_{\mathrm{def}}^{\mathrm{s} \sigma \mathrm{h}(0)}\right\rangle=\frac{1}{\sqrt{n_{\sigma}}} \sum_{i} f_{i}^{\mathrm{s} \sigma \mathrm{h}} b_{i, \sigma}\left|\Psi_{\mathrm{Mott}}^{\mathrm{ins}(0)}\right\rangle
\end{gathered}
$$

where $f_{i}^{\mathrm{s} \sigma \mathrm{p}}=f_{i}^{\mathrm{s} \sigma \mathrm{h}}$ is the eigenvector of the hopping matrix $t_{i j, \sigma}$ with the highest eigenvalue (which is $z t_{\sigma}$ with $z=2 d$ ) such that $\sum_{j} t_{i j, \sigma} f_{j}^{\mathrm{s} \sigma \mathrm{p}}=z t_{\sigma} f_{i}^{\mathrm{s} \sigma \mathrm{p}}$. The normalization condition requires that $\sum_{i}\left|f_{i}^{\mathrm{s} \sigma \mathrm{p}}\right|^{2}=1$. Notice that we choose the highest eigenvalue of $t_{i j, \sigma}$ because the hopping matrix enters the Hamiltonian as $-t_{i j, \sigma}$, and we ultimately want the lowest-energy states.

However, when the elementary excitations involve two particles (exactly one extra boson of each species) or two holes (exactly one less boson of each species), the degeneracy is lifted at second order in $t_{\uparrow}$ and $t_{\downarrow}$. Such elementary excitations occur when $U_{\uparrow \downarrow}$ is sufficiently large and attractive [26], and the wave functions (to zeroth order in $t_{\uparrow}$ and $t_{\downarrow}$ ) for the two-particle and two-hole defect states can be written as

$$
\begin{gathered}
\left|\Psi_{\mathrm{def}}^{\mathrm{tp}(0)}\right\rangle=\frac{1}{\sqrt{\left(n_{\uparrow}+1\right)\left(n_{\downarrow}+1\right)}} \sum_{i} f_{i}^{\mathrm{tp}} b_{i, \uparrow}^{\dagger} b_{i, \downarrow}^{\dagger}\left|\Psi_{\mathrm{Mott}}^{\mathrm{ins}(0)}\right\rangle, \\
\left|\Psi_{\mathrm{def}}^{\mathrm{th}(0)}\right\rangle=\frac{1}{\sqrt{n_{\uparrow} n_{\downarrow}}} \sum_{i} f_{i}^{\mathrm{th}} b_{i, \uparrow} b_{i, \downarrow}\left|\Psi_{\mathrm{Mott}}^{\mathrm{ins}(0)}\right\rangle,
\end{gathered}
$$

where $f_{i}^{\mathrm{tp}}=f_{i}^{\mathrm{th}}$ turns out to be the eigenvector of the $t_{i j, \uparrow} t_{i j, \downarrow}$ matrix with the highest eigenvalue (which is $z t_{\uparrow} t_{\downarrow}$ with $z=2 d$ ) such that $\sum_{j} t_{i j, \uparrow} t_{i j, \downarrow} f_{j}^{\mathrm{tp}}=z t_{\uparrow} t_{\downarrow} f_{i}^{\mathrm{tp}}$. Since the elementary excitations involve two particles or two holes, the degenerate defect states cannot be connected by one hopping, but rather require two hoppings to be connected. Therefore, one expects the degeneracy to be lifted at least at second order in $t_{\uparrow}$ and $t_{\downarrow}$, as discussed next.

\section{B. Ground-state energies}

Next, we employ the many-body version of the RayleighSchrödinger perturbation theory in $t_{\uparrow}$ and $t_{\downarrow}$ with respect to the ground state of the system when $t_{\uparrow}=t_{\downarrow}=0$, and calculate the energy of the Mott phase and of its defect states. The energy of the Mott state is obtained via nondegenerate perturbation 
theory, and to third order in $t_{\uparrow}$ and $t_{\downarrow}$ it is given by

$$
\begin{aligned}
\frac{E_{\mathrm{Mott}}^{\mathrm{ins}}}{M}= & \sum_{\sigma} \frac{U_{\sigma \sigma}}{2} n_{\sigma}\left(n_{\sigma}-1\right)+U_{\uparrow \downarrow} n_{\uparrow} n_{\downarrow}-\sum_{\sigma} \mu_{\sigma} n_{\sigma} \\
& -\sum_{\sigma} n_{\sigma}\left(n_{\sigma}+1\right) \frac{z t_{\sigma}^{2}}{U_{\sigma \sigma}}+O\left(t^{4}\right) .
\end{aligned}
$$

This is an extensive quantity (i.e., $E_{\text {Mott }}^{\text {ins }}$ is proportional to the number of lattice sites $M$ ). The odd-order terms in $t_{\uparrow}$ and $t_{\downarrow}$ vanish for the $d$-dimensional hypercubic lattices considered in this manuscript, which is simply because the Mott state given in Eq. (2) cannot be connected to itself by only one hopping, but rather requires two hoppings to be connected. Notice that Eq. (7) recovers the known result for the single-species $\mathrm{BH}$ model when one of the pseudospin components have vanishing filling (e.g., $\left.n_{\downarrow}=0[19,24]\right)$.

The calculation of the defect-state energies is more involved since it requires using degenerate perturbation theory. As mentioned previously, when the elementary excitations involve a single- $\sigma$-particle or a single- $\sigma$-hole, the degeneracy is lifted at first order in $t_{\uparrow}$ and $t_{\downarrow}$. A lengthy but straightforward calculation leads to the energy of the single- $\sigma$-particle defect state up to third order in $t_{\uparrow}$ and $t_{\downarrow}$ as

$$
\begin{aligned}
E_{\mathrm{def}}^{\mathrm{s} \sigma \mathrm{p}}= & E_{\mathrm{Mott}}^{\mathrm{ins}}+U_{\uparrow \downarrow} n_{-\sigma}+U_{\sigma \sigma} n_{\sigma}-\mu_{\sigma}-\left(n_{\sigma}+1\right) z t_{\sigma} \\
& -n_{\sigma}\left[\frac{n_{\sigma}+2}{2}+\left(n_{\sigma}+1\right)(z-3)\right] \frac{z t_{\sigma}^{2}}{U_{\sigma \sigma}} \\
& -2 n_{-\sigma}\left(n_{-\sigma}+1\right) \frac{U_{\uparrow \downarrow}^{2}}{U_{-\sigma-\sigma}^{2}-U_{\uparrow \downarrow}^{2}} \frac{z t_{-\sigma}^{2}}{U_{-\sigma-\sigma}} \\
& -n_{\sigma}\left(n_{\sigma}+1\right)\left[n_{\sigma}(z-1)^{2}+\left(n_{\sigma}+1\right)(z-1)(z-4)\right. \\
& \left.+\left(n_{\sigma}+2\right)(3 z / 4-1)\right] \frac{z t_{\sigma}^{3}}{U_{\sigma \sigma}^{2}}-4\left(n_{\sigma}+1\right) n_{-\sigma}\left(n_{-\sigma}+1\right) \\
& \times \frac{U_{\uparrow \downarrow}^{2}}{U_{-\sigma-\sigma}^{2}-U_{\uparrow \downarrow}^{2}}\left(z-1-\frac{U_{-\sigma-\sigma}^{2}}{U_{-\sigma-\sigma}^{2}-U_{\uparrow \downarrow}^{2}}\right) \\
& \times \frac{z t_{\sigma} t_{-\sigma}^{2}}{U_{-\sigma-\sigma}^{2}}+O\left(t^{4}\right),
\end{aligned}
$$

where $(-\uparrow) \equiv \downarrow$ and vice versa. Here, we assume $U_{\sigma \sigma} \gg$ $t_{\sigma}$ and $\left\{U_{-\sigma-\sigma},\left|U_{-\sigma-\sigma} \pm U_{\uparrow \downarrow}\right|\right\} \gg t_{-\sigma}$. Equation (8) is valid for all $d$-dimensional hypercubic lattices, and it recovers the known result for the single-species BH model when $n_{-\sigma}=0$ $[19,24]$. Note that this expression also recovers the known result for the single-species $\mathrm{BH}$ model when $U_{\uparrow \downarrow}=0$, which provides an independent check of the algebra. To third order in $t_{\uparrow}$ and $t_{\downarrow}$, we obtain a similar expression for the energy of the single- $\sigma$-hole defect state given by

$$
\begin{aligned}
E_{\mathrm{def}}^{\mathrm{s} \sigma \mathrm{h}}= & E_{\mathrm{Mott}}^{\mathrm{ins}}-U_{\uparrow \downarrow} n_{-\sigma}-U_{\sigma \sigma}\left(n_{\sigma}-1\right)+\mu_{\sigma}-n_{\sigma} z t_{\sigma} \\
& -\left(n_{\sigma}+1\right)\left[\frac{n_{\sigma}-1}{2}+n_{\sigma}(z-3)\right] \frac{z t_{\sigma}^{2}}{U_{\sigma \sigma}} \\
& -2 n_{-\sigma}\left(n_{-\sigma}+1\right) \frac{U_{\uparrow \downarrow}^{2}}{U_{-\sigma-\sigma}^{2}-U_{\uparrow \downarrow}^{2}} \frac{z t_{-\sigma}^{2}}{U_{-\sigma-\sigma}} \\
& -n_{\sigma}\left(n_{\sigma}+1\right)\left[\left(n_{\sigma}+1\right)(z-1)^{2}+n_{\sigma}(z-1)(z-4)\right.
\end{aligned}
$$

$$
\begin{aligned}
& \left.+\left(n_{\sigma}-1\right)(3 z / 4-1)\right] \frac{z t_{\sigma}^{3}}{U_{\sigma \sigma}^{2}}-4 n_{\sigma} n_{-\sigma}\left(n_{-\sigma}+1\right) \\
& \times \frac{U_{\uparrow \downarrow}^{2}}{U_{-\sigma-\sigma}^{2}-U_{\uparrow \downarrow}^{2}}\left(z-1-\frac{U_{-\sigma-\sigma}^{2}}{U_{-\sigma-\sigma}^{2}-U_{\uparrow \downarrow}^{2}}\right) \\
& \times \frac{z t_{\sigma} t_{-\sigma}^{2}}{U_{-\sigma-\sigma}^{2}}+O\left(t^{4}\right),
\end{aligned}
$$

which is also valid for all $d$-dimensional hypercubic lattices, and it also recovers the known result for the single-species BH model when $n_{-\sigma}=0$ or $U_{\uparrow \downarrow}=0[19,24]$. Here, we again assume $U_{\sigma \sigma} \gg t_{\sigma}$ and $\left\{U_{-\sigma-\sigma},\left|U_{-\sigma-\sigma} \pm U_{\uparrow \downarrow}\right|\right\} \gg t_{-\sigma}$. We also checked the accuracy of the second-order terms in Eqs. (8) and (9) via exact small-cluster (two-site) calculations with one $\sigma$ and two $-\sigma$ particles.

We note that the mean-field phase boundary between the Mott phase and its single- $\sigma$-particle and single- $\sigma$-hole defect states can be calculated as

$$
\begin{aligned}
\mu_{\sigma}^{\mathrm{par}, \mathrm{hol}}= & U_{\sigma \sigma}\left(n_{\sigma}-1 / 2\right)+U_{\uparrow \downarrow} n_{-\sigma}-z t_{\sigma} / 2 \\
& \pm \sqrt{U_{\sigma \sigma}^{2} / 4-U_{\sigma \sigma}\left(n_{\sigma}+1 / 2\right) z t_{\sigma}+z^{2} t_{\sigma}^{2} / 4}
\end{aligned}
$$

This expression is exact for infinite-dimensional hypercubic lattices, and it recovers the known result for the single-species BH model when $n_{-\sigma}=0$ or $U_{\uparrow \downarrow}=0$ [1]. In the $d \rightarrow \infty$ limit (while keeping $d t_{\sigma}$ constant), we checked that our strongcoupling perturbation results given in Eqs. (8) and (9) agree with this exact solution when the latter is expanded out to third order in $t_{\uparrow}$ and $t_{\downarrow}$, providing an independent check of the algebra. Equation (10) also shows that, for infinite-dimensional lattices, the Mott lobes are separated by $U_{\uparrow \downarrow} n_{-\sigma}$, but their shapes and critical points (the latter are obtained by setting $\mu_{\sigma}^{\mathrm{par}}=\mu_{\sigma}^{\mathrm{hol}}$ ) are independent of $U_{\uparrow \downarrow}$. This is not the case for finite-dimensional lattices as can be clearly seen from our results. It is also important to mention here that both the shapes and critical points are independent of the sign of $U_{\uparrow \downarrow}$ in finite dimensions (at the third order presented here) as can be seen in Eqs. (8) and (9).

However, when the elementary excitations involve two particles or two holes (which occurs when $U_{\uparrow \downarrow}$ is sufficiently large and attractive [26]), the degeneracy is lifted at second order in $t_{\uparrow}$ and $t_{\downarrow}$. A lengthy but straightforward calculation leads to the energy of the two-particle defect state up to third order in $t_{\uparrow}$ and $t_{\downarrow}$ as

$$
\begin{aligned}
E_{\mathrm{def}}^{\mathrm{tp}}= & E_{\mathrm{Mott}}^{\mathrm{ins}}+U_{\uparrow \downarrow}\left(n_{\uparrow}+n_{\downarrow}+1\right)+\sum_{\sigma}\left(U_{\sigma \sigma} n_{\sigma}+\mu_{\sigma}\right) \\
& +\frac{2\left(n_{\uparrow}+1\right)\left(n_{\downarrow}+1\right)}{U_{\uparrow \downarrow}} z t_{\uparrow} t_{\downarrow}+\sum_{\sigma}\left[\frac{\left(n_{\sigma}+1\right)^{2}}{U_{\uparrow \downarrow}}\right. \\
& \left.-\frac{n_{\sigma}\left(n_{\sigma}+2\right)}{2 U_{\sigma \sigma}+U_{\uparrow \downarrow}}+\frac{2 n_{\sigma}\left(n_{\sigma}+1\right)}{U_{\sigma \sigma}}\right] z t_{\sigma}^{2}+O\left(t^{4}\right) .
\end{aligned}
$$

Here, we assume $\left\{U_{\sigma \sigma},\left|U_{\uparrow \downarrow}\right|, 2 U_{\sigma \sigma}+U_{\uparrow \downarrow}\right\} \gg t_{\sigma}$. Equation (11) is valid for all $d$-dimensional hypercubic lattices, where the odd-order terms in $t_{\uparrow}$ and $t_{\downarrow}$ vanish [27]. To third order in $t_{\uparrow}$ and $t_{\downarrow}$, we obtain a similar expression for the energy of the 
two-hole defect state given by

$$
\begin{aligned}
E_{\mathrm{def}}^{\mathrm{th}}= & E_{\mathrm{Mott}}^{\mathrm{ins}}-U_{\uparrow \downarrow}\left(n_{\uparrow}+n_{\downarrow}-1\right)-\sum_{\sigma}\left[U_{\sigma \sigma}\left(n_{\sigma}-1\right)-\mu_{\sigma}\right] \\
& +\frac{2 n_{\uparrow} n_{\downarrow}}{U_{\uparrow \downarrow}} z t_{\uparrow} t_{\downarrow}+\sum_{\sigma}\left[\frac{n_{\sigma}^{2}}{U_{\uparrow \downarrow}}-\frac{\left(n_{\sigma}^{2}-1\right)}{2 U_{\sigma \sigma}+U_{\uparrow \downarrow}}\right. \\
& \left.+\frac{2 n_{\sigma}\left(n_{\sigma}+1\right)}{U_{\sigma \sigma}}\right] z t_{\sigma}^{2}+O\left(t^{4}\right),
\end{aligned}
$$

which is also valid for all $d$-dimensional hypercubic lattices, where the odd-order terms in $t_{\uparrow}$ and $t_{\downarrow}$ vanish [27]. Here, we again assume $\left\{U_{\sigma \sigma},\left|U_{\uparrow \downarrow}\right|, 2 U_{\sigma \sigma}+U_{\uparrow \downarrow}\right\} \gg t_{\sigma}$. Since the single- $\sigma$-particle and single- $\sigma$-hole defect states have corrections to first order in the hopping, while the two-particle and two-hole defect states have corrections to second order in the hopping, the slopes of the Mott lobes are finite as $\left\{t_{\uparrow}, t_{\downarrow}\right\} \rightarrow 0$ in the former case, but they vanish in the latter case. Hence, the shape of the insulating lobes are expected to be very different for two-particle or two-hole excitations. In addition, the chemical-potential widths $\left(\mu_{\sigma}\right)$ of all Mott lobes are $U_{\sigma \sigma}$ in the former case, but they $\left[\left(\mu_{\uparrow}+\mu_{\downarrow}\right) / 2\right]$ are $U_{\uparrow \downarrow}+\left(U_{\uparrow \uparrow}+U_{\downarrow \downarrow}\right) / 2$ in the latter.

We note that in the limit when $t_{\uparrow}=t_{\downarrow}=t, U_{\uparrow \uparrow}=U_{\downarrow \downarrow}=$ $U_{0}, U_{\uparrow \downarrow}=U^{\prime}, n_{\uparrow}=n_{\downarrow}=n_{0}, \mu_{\uparrow}=\mu_{\downarrow}=\mu$, and $z=2$ (or $d=1$ ), Eq. (12) is in complete agreement with Eq. (3) of Ref. [11], providing an independent check of the algebra. In addition, in the limit when $t_{\uparrow}=t_{\downarrow}=J, U_{\uparrow \uparrow}=U_{\downarrow \downarrow}=U$, $U_{\uparrow \downarrow}=W \approx-U, n_{\uparrow}=n_{\downarrow}=m$, and $\mu_{\uparrow}=\mu_{\downarrow}=\mu$, Eqs. (11) and (12) reduce to those given in Ref. [12] (after setting $U_{N N}=$ 0 there). However, the terms that are proportional to $t_{\uparrow} t_{\downarrow}$ are not included in their definitions of the two-particle and two-hole excitation gaps. We also checked the accuracy of Eqs. (11) and (12) via exact small-cluster (two-site) calculations with one particle of each species.

We would also like to remark in passing that the energy difference between the Mott phase and its defect states determine the phase boundary of the particle and hole branches. This is because at the point where the energy of the incompressible state becomes equal to its defect state, the system becomes compressible, assuming that the compressibility approaches zero continuously at the phase boundary. While $E_{\text {Mott }}^{\text {ins }}$ and its defects $E_{\text {def }}^{\text {sop }}, E_{\text {def }}^{\mathrm{s} \sigma \mathrm{h}}, E_{\text {def }}^{\mathrm{tp}}$, and $E_{\text {def }}^{\text {th }}$ depend on the lattice size $M$, their differences do not. Therefore, the chemical potentials that determine the particle and hole branches are independent of $M$ at the phase boundaries. This indicates that the numerical Monte Carlo simulations should not have a strong dependence on $M$.

It is known that the third-order strong-coupling expansion is not very accurate near the tip of the Mott lobes, as $t_{\uparrow}$ and $t_{\downarrow}$ are not very small there $[19,24]$. For this reason, an extrapolation technique is highly desirable to determine more accurate phase diagrams. Therefore, having discussed the third-order strong-coupling expansion for a general two-species BoseBose mixtures with arbitrary hoppings $t_{\sigma}$, interactions $U_{\sigma \sigma^{\prime}}$, densities $n_{\sigma}$, and chemical potentials $\mu_{\sigma}$, next we show how to develop a scaling theory.

\section{EXTRAPOLATION TECHNIQUE}

In this section, we propose a chemical potential extrapolation technique based on scaling theory to extrapolate our third-order power-series expansion into a functional form that is appropriate for the entire Mott lobes. It is known that the critical point at the tip of the lobes has the scaling behavior of a $(d+1)$-dimensional $X Y$ model, and therefore the lobes have Kosterlitz-Thouless shapes for $d=1$ and power-law shapes for $d>1$. For illustration purposes, here we analyze only the latter case, but this technique can be easily adapted to the $d=1$ case [19].

\section{A. Scaling ansatz}

From now on we consider a two-species mixture with $t_{\uparrow}=$ $t_{\downarrow}=t, U_{\uparrow \uparrow}=U_{\downarrow \downarrow}=U, U_{\uparrow \downarrow}=V, n_{\uparrow}=n_{\downarrow}=n$, and $\mu_{\uparrow}=$ $\mu_{\downarrow}=\mu$. When $d>1$, we propose the following ansatz which includes the known power-law critical behavior of the tip of the lobes,

$$
\frac{\mu^{ \pm}}{U}=A(x) \pm B(x)\left(x_{c}-x\right)^{z v},
$$

where $A(x)=a+b x+c x^{2}+d x^{3}+\cdots$ and $B(x)=\alpha+$ $\beta x+\gamma x^{2}+\delta x^{3}+\cdots$ are regular functions of $x=2 d t / U, x_{c}$ is the critical point which determines the location of the lobes, and $z v$ is the critical exponent for the $(d+1)$-dimensional $X Y$ model which determines the shape of the lobes near $x_{c}=2 d t_{c} / U$. In Eq. (13), the plus sign corresponds to the particle branch, and the minus sign corresponds to the hole branch. The form of the ansatz is taken to be the same for both single- and two-particle (or single- and two-hole) excitations, but the parameters are very different.

The parameters $a, b, c$, and $d$ depend on $U, V$, and $n$, and they are determined by matching them with the coefficients given by our third-order expansion such that $A(x)=\left(\mu^{\mathrm{par}}+\mu^{\mathrm{hol}}\right) /(2 U)$. Here, $\mu^{\mathrm{par}}$ and $\mu^{\text {hol }}$ are our strong-coupling expansion results determined from Eqs. (8) and (9) for the single-particle and single-hole excitations, or from Eqs. (11) and (12) for the two-particle and twohole excitations, respectively. Writing our strong-coupling expansion results for the particle and hole branches in the form $\mu^{\mathrm{par}}=U \sum_{n=0}^{3} e_{n}^{+} x^{n}$ and $\mu^{\mathrm{hol}}=U \sum_{n=0}^{3} e_{n}^{-} x^{n}$, leads to $a=\left(e_{0}^{+}+e_{0}^{-}\right) / 2, b=\left(e_{1}^{+}+e_{1}^{-}\right) / 2, c=\left(e_{2}^{+}+e_{2}^{-}\right) / 2$, and $d=\left(e_{3}^{+}+e_{3}^{-}\right) / 2$. To determine the $U, V$, and $n$ dependence of the parameters $\alpha, \beta, \gamma, \delta, x_{c}$, and $z \nu$, we first expand the left-hand side of $B(x)\left(x_{c}-x\right)^{z v}=\left(\mu^{\text {par }}-\mu^{\text {hol }}\right) /(2 U)$ in powers of $x$, and match the coefficients with the coefficients given by our third-order expansion, leading to

$$
\begin{gathered}
\alpha=\frac{e_{0}^{+}-e_{0}^{-}}{2 x_{c}^{z v}}, \\
\frac{\beta}{\alpha}=\frac{z v}{x_{c}}+\frac{e_{1}^{+}-e_{1}^{-}}{e_{0}^{+}-e_{0}^{-}}, \\
\frac{\gamma}{\alpha}=\frac{z v(z v+1)}{2 x_{c}^{2}}+\frac{z v}{x_{c}} \frac{e_{1}^{+}-e_{1}^{-}}{e_{0}^{+}-e_{0}^{-}}+\frac{e_{2}^{+}-e_{2}^{-}}{e_{0}^{+}-e_{0}^{-}}, \\
\frac{\delta}{\alpha}=\frac{z v(z v+1)(z v+2)}{6 x_{c}^{3}}+\frac{z v(z v+1)}{2 x_{c}^{2}} \frac{e_{1}^{+}-e_{1}^{-}}{e_{0}^{+}-e_{0}^{-}} \\
+\frac{z v}{x_{c}} \frac{e_{2}^{+}-e_{2}^{-}}{e_{0}^{+}-e_{0}^{-}}+\frac{e_{3}^{+}-e_{3}^{-}}{e_{0}^{+}-e_{0}^{-}} .
\end{gathered}
$$


We fix $z v$ at its well-known values such that $z v \approx 2 / 3$ for $d=2$ and $z v=1 / 2$ for $d>2$. If the exact value of $x_{c}$ is known via other means (e.g., numerical simulations), $\alpha, \beta, \gamma$, and $\delta$ can be calculated accordingly, for which the extrapolation technique gives very accurate results $[23,25]$. If the exact value of $x_{c}$ is not known, then we set $\delta=0$, and solve Eqs. (14)-(16) and the $\delta=0$ equation to determine $\alpha, \beta, \gamma$, and $x_{c}$ selfconsistently, which also leads to accurate results [19,24]. Next we present typical ground-state phase diagrams for $(d=2)$ and $(d=3)$-dimensional hypercubic lattices obtained from this extrapolation technique.

\section{B. Numerical results}

In Figs. 1 and 2, the results of the third-order strongcoupling expansion (dotted lines) are compared to those of the extrapolation technique (hollow pink squares and solid black circles) when $V=0.5 U$ and $V=-0.85 U$, respectively, in two $(d=2$ or $z=4)$ and three $(d=3$ or $z=6)$ dimensions. We recall here that $t_{\uparrow}=t_{\downarrow}=t, U_{\uparrow \uparrow}=U_{\downarrow \downarrow}=U, U_{\uparrow \downarrow}=V$, $n_{\uparrow}=n_{\downarrow}=n$, and $\mu_{\uparrow}=\mu_{\downarrow}=\mu$.

In Fig. 1, we show the chemical potential $\mu$ (in units of $U$ ) versus $x=2 d t / U$ phase diagram for (a) two-dimensional and (b) three-dimensional hypercubic lattices, where we choose the interspecies interaction to be repulsive $V=0.5 U$. Comparing Eqs. (8) and (9) with Eqs. (11) and (12), we expect the excited state of the system to be the usual superfluid for all $V>0$ for all $t$. The dotted lines correspond to phase boundary for the Mott insulator to superfluid state as determined from the thirdorder strong-coupling expansion, and the hollow pink squares correspond to the extrapolation fits for the single-particle and single-hole excitations discussed in the text. We recall here that an incompressible supercounter flow phase $[7-9,13]$ also exists outside of the Mott insulator lobes, but our current formalism cannot be used to locate its phase boundary.

At $t=0$, the chemical potential width of all Mott lobes are $U$ (similar to the single-species $\mathrm{BH}$ model), but they are separated from each other by $V$ as a function of $\mu$. As $t$ increases from zero, the range of $\mu$ about which the ground state is a Mott insulator decreases, and the Mott insulator phase disappears at a critical value of $t$, beyond which the system becomes a superfluid. In addition, similar to what was found for the single-species BH model [19,24], the strongcoupling expansion overestimates the phase boundaries, and it leads to unphysical pointed tips for all Mott lobes, which is expected since a finite-order expansion cannot describe the physics of the critical point correctly. A short list of $V / U$ versus the critical points $x_{c}=2 d t_{c} / U$ is presented for the first two Mott insulator lobes in Table I, where it is shown that the critical points tend to move in as $V$ increases. This is because the presence of a second species (say $-\sigma$ ones) screens the onsite intraspecies repulsion $U_{\sigma \sigma}$ between $\sigma$ species and, hence, increases the superfluid region.

In Fig. 2, we show the chemical potential $\mu$ (in units of $U$ ) versus $x=2 d t / U$ phase diagram for (a) two-dimensional and (b) three-dimensional hypercubic lattices, where in these figures we choose the interspecies interaction to be attractive $V=-0.85 U$. Comparing Eqs. (8) and (9) with Eqs. (11) and (12), we expect the excited state of the system to be a paired superfluid for all $V<0$ when $t \rightarrow 0$. This is clearly
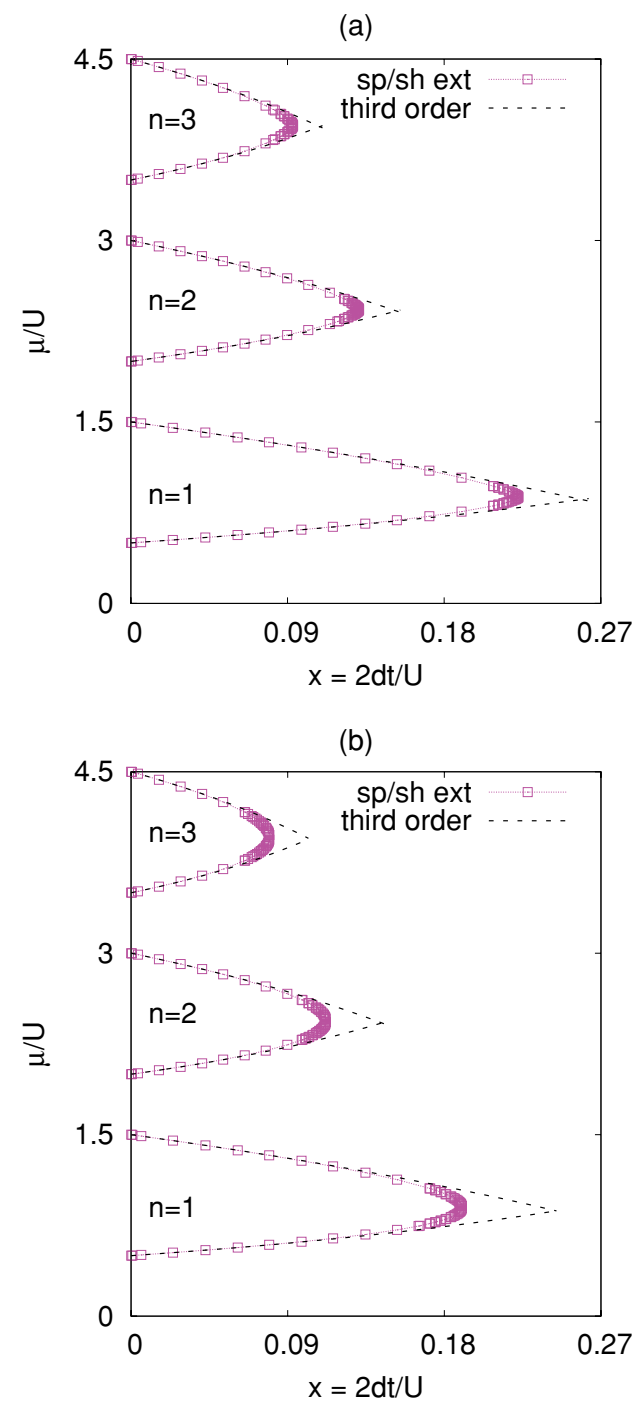

FIG. 1. (Color online) Chemical potential $\mu$ (in units of $U$ ) versus $x=2 d t / U$ phase diagram for (a) two- and (b) three-dimensional hypercubic lattices with $t_{\uparrow}=t_{\downarrow}=t, U_{\uparrow \uparrow}=U_{\downarrow \downarrow}=U, U_{\uparrow \downarrow}=V=$ $0.5 U, n_{\uparrow}=n_{\downarrow}=n$, and $\mu_{\uparrow}=\mu_{\downarrow}=\mu$. The dotted lines correspond to the phase boundary for the Mott insulator to superfluid state as determined from the third-order strong-coupling expansion, and the hollow pink squares to the extrapolation fit for the single-particle or single-hole excitations discussed in the text. Recall that an incompressible supercounter flow phase also exists outside of the Mott insulator lobes.

seen in the figure where the dotted lines correspond to phase boundary for the Mott insulator to superfluid state as determined from the third-order strong-coupling expansion, the hollow pink squares correspond to the extrapolation fits for the single-particle and single-hole excitations (shown only for illustration purposes), and the solid black circles correspond to the extrapolation fits for the two-particle and two-hole excitations (this is the expected transition) discussed in the text.

At $t=0$, the chemical potential width of all Mott lobes are $V+U=0.15 U$, which is in contrast with the single-species $\mathrm{BH}$ model. As $t$ increases from zero, the range of $\mu$ about which the ground state is a Mott insulator decreases here as 
(a)

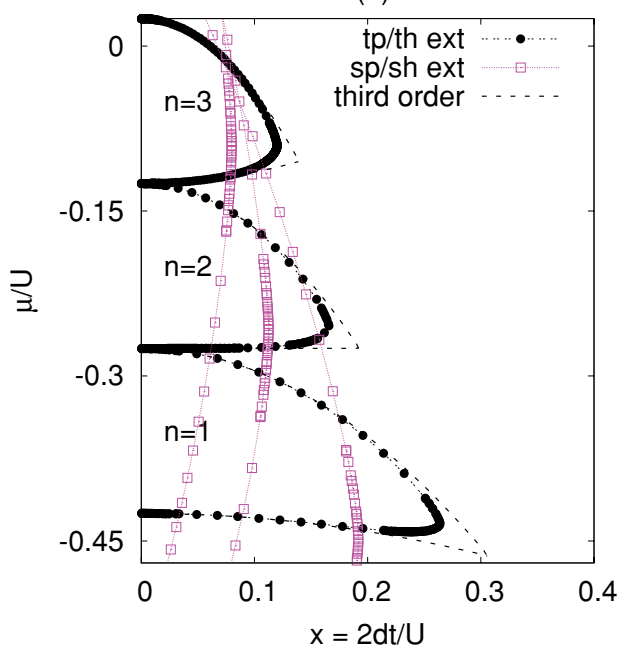

(b)

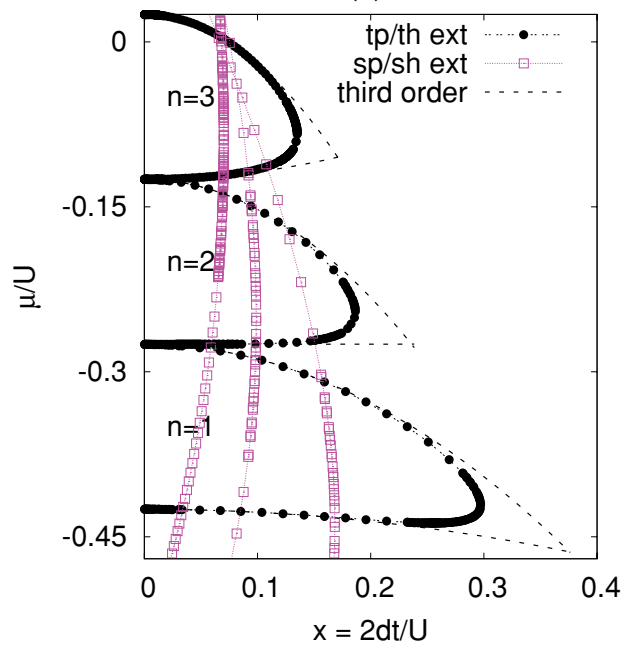

FIG. 2. (Color online) Chemical potential $\mu$ (in units of $U$ ) versus $x=2 d t / U$ phase diagram for (a) two- and (b) threedimensional hypercubic lattices with $t_{\uparrow}=t_{\downarrow}=t, U_{\uparrow \uparrow}=U_{\downarrow \downarrow}=U$, $U_{\uparrow \downarrow}=V=-0.85 U, n_{\uparrow}=n_{\downarrow}=n$, and $\mu_{\uparrow}=\mu_{\downarrow}=\mu$. The dotted lines correspond to the phase boundary for the Mott insulator to superfluid state determined from the third-order strong-coupling expansion, the hollow pink squares to the extrapolation fit for the single-particle or single-hole excitations (shown only for illustration purposes), and the solid black circles to the extrapolation fit for the two-particle or two-hole excitations (the expected transition) discussed in the text.

well, and the Mott insulator phase disappears at a critical value of $t$, beyond which the system becomes a paired superfluid. The strong-coupling expansion again overestimates the phase boundaries, and it again leads to unphysical pointed tips for all Mott lobes. In addition, a short list of $V / U$ versus the critical points $x_{c}=2 d t_{c} / U$ are presented for the first two Mott insulator lobes in Table II. Our results are consistent with the expectation that, for small $V$, the locations of the tips increase as a function of $V$, because the presence of a nonzero $V$ is what allowed these states to form in the first place. However, when $V$ is larger than some critical value $(\sim 0.6 U)$, the locations of the tips decrease, and they eventually vanish when $V=-U$.
TABLE I. List of the critical points (location of the tips) $x_{c}=$ $2 d t_{c} / U$ for the first two Mott insulator lobes that are found from the chemical potential extrapolation technique described in the text. Here, $t_{\uparrow}=t_{\downarrow}=t, U_{\uparrow \uparrow}=U_{\downarrow \downarrow}=U, U_{\uparrow \downarrow}=V, n_{\uparrow}=n_{\downarrow}=n$, and $\mu_{\uparrow}=\mu_{\downarrow}=\mu$. These critical points for the single-particle or single-hole excitations are determined from Eqs. (8) and (9), and they tend to move in as $V$ increases, and are independent of the sign of $V$.

\begin{tabular}{llllll}
\hline \hline & \multicolumn{2}{c}{$d=2$} & & \multicolumn{2}{c}{$d=3$} \\
\cline { 2 - 3 }$V / U$ & $n=1$ & $n=2$ & & $n=1$ & $n=2$ \\
\hline 0.0 & 0.234 & 0.138 & & 0.196 & 0.116 \\
0.1 & 0.234 & 0.138 & & 0.196 & 0.115 \\
0.2 & 0.233 & 0.137 & & 0.195 & 0.115 \\
0.3 & 0.230 & 0.136 & & 0.194 & 0.114 \\
0.4 & 0.227 & 0.134 & & 0.193 & 0.113 \\
0.5 & 0.223 & 0.131 & & 0.190 & 0.112 \\
0.6 & 0.217 & 0.128 & 0.187 & 0.110 \\
0.7 & 0.208 & 0.123 & 0.182 & 0.107 \\
0.8 & 0.197 & 0.116 & 0.174 & 0.102 \\
0.9 & 0.193 & 0.113 & 0.163 & 0.095 \\
\hline \hline
\end{tabular}

This may indicate an instability toward a collapse since at this point $U_{\uparrow \uparrow} U_{\downarrow \downarrow}$ is exactly equal to $U_{\uparrow \downarrow}^{2}$.

Compared to the $V>0$ case shown in Fig. 1, note that the shape of the Mott insulator to paired superfluid phase boundary is very different, showing a re-entrant behavior in

TABLE II. List of the critical points (location of the tips) $x_{c}=2 d t_{c} / U$ that are found from the chemical potential extrapolation technique described in the text. Here, $t_{\uparrow}=t_{\downarrow}=t, U_{\uparrow \uparrow}=U_{\downarrow \downarrow}=U$, $U_{\uparrow \downarrow}=V, n_{\uparrow}=n_{\downarrow}=n$, and $\mu_{\uparrow}=\mu_{\downarrow}=\mu$. These critical points for the two-particle or two-hole excitations are determined from Eqs. (11) and (12) when $V<0$. Note that, for small $V, x_{c}$ 's tend to increase as a function of $V$, since the presence of a nonzero $V$ is what allowed these states to form in the first place. However, $x_{c}$ 's decrease beyond a critical $V$, and they eventually vanish when $V=-U$, which may indicate an instability toward a collapse.

\begin{tabular}{llllll}
\hline \hline & \multicolumn{2}{c}{$d=2$} & & \multicolumn{2}{c}{$d=3$} \\
\cline { 2 - 3 }$V / U$ & $n=1$ & $n=2$ & & $n=1$ & $n=2$ \\
\hline-0.01 & 0.0543 & 0.0337 & & 0.0611 & 0.0379 \\
-0.03 & 0.0937 & 0.0582 & & 0.105 & 0.0655 \\
-0.05 & 0.121 & 0.0749 & & 0.136 & 0.0843 \\
-0.07 & 0.142 & 0.0883 & & 0.160 & 0.0994 \\
-0.1 & 0.169 & 0.105 & & 0.190 & 0.118 \\
-0.2 & 0.233 & 0.145 & & 0.262 & 0.164 \\
-0.3 & 0.277 & 0.173 & & 0.311 & 0.195 \\
-0.4 & 0.307 & 0.193 & & 0.345 & 0.217 \\
-0.5 & 0.325 & 0.205 & & 0.366 & 0.230 \\
-0.6 & 0.331 & 0.209 & & 0.372 & 0.235 \\
-0.7 & 0.321 & 0.203 & 0.362 & 0.228 \\
-0.8 & 0.291 & 0.183 & 0.327 & 0.206 \\
-0.9 & 0.225 & 0.141 & 0.253 & 0.159 \\
-0.93 & 0.193 & 0.121 & 0.217 & 0.136 \\
-0.95 & 0.166 & 0.103 & 0.187 & 0.116 \\
-0.97 & 0.1304 & 0.0812 & 0.147 & 0.0913 \\
-0.99 & 0.0764 & 0.0474 & 0.0860 & 0.0534 \\
\hline \hline
\end{tabular}


all dimensions from paired superfluid to Mott insulator and again to a paired superfluid phase, as a function of $t$. Our results are consistent with an early numerical time-evolving block decimation (TEBD) calculation [11], where such a re-entrant quantum phase transition in one dimension was predicted.

The re-entrant quantum phase transition occurs when the coefficient of the hopping term in Eq. (12) is negative [so that the two-hole excitation branch has a negative slope in $\left(\mu_{\uparrow}+\mu_{\downarrow}\right) / 2$ versus $t_{\sigma}$ phase diagram when $\left.t_{\sigma} \rightarrow 0\right]$, that is, $-\left(2 n_{\uparrow} n_{\downarrow} / U_{\uparrow \downarrow}\right) z t_{\uparrow} t_{\downarrow}-\sum_{\sigma}\left[n_{\sigma}^{2} / U_{\uparrow \downarrow}-\left(n_{\sigma}^{2}-1\right) /\left(2 U_{\sigma \sigma}+\right.\right.$ $\left.\left.U_{\uparrow \downarrow}\right)+2 n_{\sigma}\left(n_{\sigma}+1\right) / U_{\sigma \sigma}\right] z t_{\sigma}^{2}$ term, which occurs for the first few Mott lobes beyond a critical $U_{\uparrow \downarrow}$. When this coefficient is negative, its value is most negative for the first Mott lobe, and therefore the effect is strongest there. However, the coefficient increases and eventually becomes positive as a function of filling, and thus the re-entrant behavior becomes weaker as filling increases, and it eventually disappears beyond a critical filling. For the parameters used in Fig. 2, this occurs only for the first lobe, as can be seen in the figures. We also note that the sign of this coefficient is independent of the dimensionality of the lattice, since $z=2 d$ enters into the coefficient only as an overall factor.

What happens when $t_{\uparrow} \neq t_{\downarrow}$ and/or $U_{\uparrow \uparrow} \neq U_{\downarrow \downarrow}$ ? We do not expect any qualitative change for attractive interspecies interactions. However, for repulsive interspecies interactions, this lifts the degeneracy of the single-particle or single-hole excitation energies. While the transition is from a doubleMott insulator to a double superfluid of both species in the degenerate case, it is from a double-Mott insulator of both species to a Mott insulator of one species and a superfluid of the other in the nondegenerate case.

\section{v. CONCLUSIONS}

We analyzed the zero temperature phase diagram of the twospecies Bose-Hubbard (BH) model with onsite boson-boson interactions in $d$-dimensional hypercubic lattices, including both the repulsive and attractive interspecies interaction. We used the many-body version of the Rayleigh-Schrödinger perturbation theory in the kinetic energy term with respect to the ground state of the system when the kinetic energy term is absent, and calculate ground-state energies needed to carry out our analysis. This technique was previously used to discuss the phase diagram of the single-species BH model [19-21,23], the extended $\mathrm{BH}$ model [24], and the hardcore $\mathrm{BH}$ model with a superlattice [25], and its results showed an excellent agreement with Monte Carlo simulations [23,25]. Motivated by the success of this technique with these models, here we generalized it to the two-species BH model, hoping to develop an analytical approach which could be as accurate as the numerical ones.

We derived analytical expressions for the phase boundary between the incompressible Mott insulator and the compressible superfluid phase up to third order in the hoppings. We also proposed a chemical potential extrapolation technique based on the scaling theory to extrapolate our third-order power series expansion into a functional form that is appropriate for the Mott lobes. In particular, when the interspecies interaction is sufficiently large and attractive, we found a re-entrant quantum phase transition from paired superfluid (superfluidity of composite bosons, i.e., Bose-Bose pairs) to Mott insulator and again to a paired superfluid in all one, two, and three dimensions. Since the available Monte Carlo calculations $[9,10]$ do not provide the Mott insulator to superfluid transition phase boundary in the experimentally more relevant chemical potential versus hopping plane, we could not compare our results with them. This comparison is highly desirable to judge the accuracy of our strong-coupling expansion results.

A possible direction to extend this work is to consider the limit where hopping of one species is much larger than the other. In this limit, the two-species $\mathrm{BH}$ model reduces to the Bose-Bose version of the Falicov-Kimball model [28], the Fermi-Fermi version of which has been widely discussed in the condensed-matter literature and the Fermi-Bose version has just been studied [29]. It is known for such models that there is a tendency toward both phase separation and density wave order [30], which requires a new calculation partially similar to that of Ref. [24]. One can also examine how the momentum distribution changes with the hopping in the insulating phases $[23,31]$, which has direct relevance to ultracold atomic experiments.

\section{ACKNOWLEDGMENTS}

The author thanks Anzi Hu, L. Mathey, and J. K. Freericks for discussions, and The Scientific and Technological Research Council of Turkey (TÜBİTAK) for financial support.
[1] M. P. A. Fisher, P. B. Weichman, G. Grinstein, and D. S. Fisher, Phys. Rev. B 40, 546 (1989).

[2] M. Greiner, O. Mandel, T. Esslinger, T. W. Hänsch, and I. Bloch, Nature (London) 415, 39 (2002).

[3] T. Stöferle, H. Moritz, C. Schori, M. Köhl, and T. Esslinger, Phys. Rev. Lett. 92, 130403 (2004).

[4] I. B. Spielman, W. D. Phillips, and J. V. Porto, Phys. Rev. Lett. 98, 080404 (2007).

[5] I. B. Spielman, W. D. Phillips, and J. V. Porto, Phys. Rev. Lett. 100, 120402 (2008).

[6] I. Bloch, J. Dalibard, and W. Zwerger, Rev. Mod. Phys. 80, 885 (2008).
[7] A. B. Kuklov and B. V. Svistunov, Phys. Rev. Lett. 90, 100401 (2003).

[8] E. Altman, W. Hofstetter, E. Demler, and M. D. Lukin, New J. Phys. 5, 113 (2003).

[9] A. Kuklov, N. Prokof'ev, and B. Svistunov, Phys. Rev. Lett. 92, 050402 (2004).

[10] A. Isacsson, Min-Chul Cha, K. Sengupta, and S. M. Girvin, Phys. Rev. B 72, 184507 (2005).

[11] A. Argüelles and L. Santos, Phys. Rev. A 75, 053613 (2007).

[12] C. Trefzger, C. Menotti, and M. Lewenstein, Phys. Rev. Lett. 103, 035304 (2009). 
[13] A. Hu, L. Mathey, I. Danshita, E. Tiesinga, C. J. Williams, and C. W. Clark, Phys. Rev. A 80, 023619 (2009).

[14] P. Buonsante, S. M. Giampaolo, F. Illuminati, V. Penna, and A. Vezzani, Eur. Phys. J. B 68, 427 (2009).

[15] A. Hubener, M. Snoek, and W. Hofstetter, Phys. Rev. B 80, 245109 (2009).

[16] C. Menotti and S. Stringari, Phys. Rev. A 81, 045604 (2010).

[17] J. Catani, L. De Sarlo, G. Barontini, F. Minardi, and M. Inguscio, Phys. Rev. A 77, 011603(R) (2008).

[18] G. Thalhammer, G. Barontini, L. De Sarlo, J. Catani, F. Minardi, and M. Inguscio, Phys. Rev. Lett. 100, 210402 (2008).

[19] J. K. Freericks and H. Monien, Phys. Rev. B 53, 2691 (1996).

[20] T. D. Kühner and H. Monien, Phys. Rev. B 58, 14741(R) (1998).

[21] P. Buonsante, V. Penna, and A. Vezzani, Phys. Rev. B 70, 184520 (2004).

[22] K. Sengupta and N. Dupuis, Phys. Rev. A 71, 033629 (2005).

[23] J. K. Freericks, H. R. Krishnamurthy, Y. Kato, N. Kawashima, and N. Trivedi, Phys. Rev. A 79, 053631 (2009).
[24] M. Iskin and J. K. Freericks, Phys. Rev. A 79, 053634 (2009).

[25] I. Hen, M. Iskin, and M. Rigol, Phys. Rev. B 81, 064503 (2010).

[26] Recall that $U_{\uparrow \downarrow}^{2}$ cannot be greater than or equal to $U_{\uparrow \uparrow} U_{\downarrow \downarrow}$, otherwise the mixture would be unstable against collapse. In addition (see, e.g., Fig. 7 in [13]), where TEBD calculations show in one dimension that $V \lesssim-0.06 U$ is already sufficient for the Mott insulator to paired superfluid transition.

[27] Note that, unlike those of single-particle and single-hole excitations where $d t_{\sigma}$ is a constant when $d \rightarrow \infty$, in the case of two-particle and two-hole excitations, $d t_{\sigma}^{2}$ must be kept constant when $d \rightarrow \infty$. In this respect, Eqs. (11) and (12) do not contain any finite- $d$ correction at the second order in hopping.

[28] L. M. Falicov and J. C. Kimball, Phys. Rev. Lett. 22, 997 (1969).

[29] M. Iskin and J. K. Freericks, Phys. Rev. A 80, 053623 (2009); and see references therein.

[30] Ş. G. Söyler, B. Capogrosso-Sansone, N. V. Prokof'ev, and B. V. Svistunov, New J. Phys. 11, 073036 (2009).

[31] M. Iskin and J. K. Freericks, Phys. Rev. A 80, 063610 (2009). 